\title{
Effect of simultaneous pancreas-kidney transplantation on blood glucose level for patients with end-stage renal disease with type 1 and type 2 diabetes
}

\author{
Luhao Liu, Yunyi Xiong, Tao Zhang, Jiali Fang, Lei Zhang, Guanghui Li, Lu Xu, Xingqiang Lai, \\ Rongxin Chen, Yuhe Guo, Guanghui Pan, Junjie Ma, Zheng Chen \\ Department of Organ Transplantation, The Second Affiliated Hospital of Guangzhou Medical University, Guangzhou 511447, China \\ Contributions: (I) Conception and design: L Liu; (II) Administrative support: G Pan, J Ma, Z Chen; (III) Provision of study materials or patients: \\ L Xu, L Zhang, X Lai, Y Guo; (IV) Collection and assembly of data: R Chen, G Li, J Fang; (V) Data analysis and interpretation: L Liu, Y Xiong, \\ T Zhang; (VI) Manuscript writing: All authors; (VII) Final approval of manuscript: All authors. \\ Correspondence to: Zheng Chen, MD, PhD. Department of Organ Transplantation, The Second Affiliated Hospital of Guangzhou Medical University, \\ Guangzhou 511447, China. Email: docchenzheng@163.com.
}

Background: Simultaneous pancreas-kidney (SPK) transplants for patients with type 1 diabetes mellitus (T1DM) remains disproportionately higher than that for type 2 diabetes mellitus (T2DM) patients. However, understanding the surgical outcomes for these patients is not well described. Therefore, the results of DM patients with end-stage renal disease and their transplantations were reported.

Methods: Between September 2016 and June 2019, 63 SPK transplants were performed in our organ transplantation center. $\chi^{2}$ and $t$-test compared the variables between the groups and the record review verified the patient survival. Using Kaplan-Meier survival estimates and Cox proportional hazards regression, we examined the influence of SPK on patient and graft survivals.

Results: Sixty-three SPK transplantation was performed, 18 (29\%) were T1DM, and 45 (71\%) T2DM. T2DM recipients had older age, duration of diabetes, and pretransplant dialysis time. No differences were found in human leukocyte antigen (HLA) mismatch, body mass index (BMI), and other variables. Patient survivals for T1DM was $98.2 \%$ and $94.8 \%$ at 1 and 2 years $v s .100 \%$ and $94.1 \%$ for T2DM recipients $(\mathrm{P}=0.87)$. There was no increased risk between kidney disease, pancreas failure, or death when comparing T2DM and T1DM.

Conclusions: In our single-center cohort of SPK transplants, we concluded that SPK recipients with T2DM diagnosis were not at increased risk for death, kidney failure, or pancreas failure when compared with recipients with T1DM.

Keywords: Diabetes mellitus (DM); kidney transplantation; simultaneous pancreas-kidney transplantation (SPK transplantation); type 2 diabetes mellitus (T2DM)

Submitted Sep 03, 2019. Accepted for publication Oct 16, 2019.

doi: 10.21037/atm.2019.10.106

View this article at: http://dx.doi.org/10.21037/atm.2019.10.106

\section{Introduction}

It is known that simultaneous pancreas-kidney (SPK) transplantation is a new and promising therapeutic option for the treatment for type 1 diabetes mellitus (T1DM) patients and end-stage renal disease. From December 16,
1966, to December 31, 2016, more than 30,000 pancreas transplants in the USA and 50,000 worldwide have been recorded on the International Pancreas Transplant Registry (IPTR) (1). From the past three decades, SPK transplantation results have been shown to be superior to pancreas transplantation, although this disparity has 
reduced over time. There is a consensus that patients with T1DM with end-stage renal disease should receive an SPK transplant if a suitable donor is available (2).

However, the clinical outcomes of SPK in type 2 diabetes mellitus (T2DM) patients are described less fully. Furthermore, there is little information available regarding the long-term glycemic control and graft survivals after SPK transplantation in T2DM patients. An impaired glucose tolerance or insulin resistance or DM recurrence probably existed in some of these patients after SPK transplantation. Thus, its value in T2DM patients continues to be carefully evaluated.

At our institution, 63 SPK transplants, with at least four months follow-up after transplantation, have been performed. Furthermore, T2DM was present in $71 \%$ of the SPK recipients' cohort. This study aimed to compare the long-term effects of pancreas transplantation about glycemic control and graft survivals between T1DM and T2DM patients.

\section{Methods}

\section{Study population}

The Institutional Review Board of the Second Affiliated Hospital of Guangzhou Medical University approved protocols of the study. Written informed consent was obtained from each participant for the collection of clinical data. This trial is registered with Chinese Clinical Trial Registry, number ChiCTR1900026543. Between September 2016 and June 2019, a total of 63 T1DM and T2DM patients with end-stage renal disease who underwent SPK were included in the present study. The recipients who had a previous transplant of a pancreas- or kidney-alone were excluded. The study population was stratified into two groups according to their diabetes diagnosis: T1DM and T2DM. T1DM is an auto-immune condition in which the body destroys the beta cells in the pancreas that produce insulin. Type 2 is a metabolic disorder where our body cells unable to use insulin effectively because the cells of the body are resistant to the action of insulin.

\section{Surgical techniques}

Renal transplants were performed to the right external iliac artery and vein, and the ureter was anastomosed to the recipient ureter by using end-to-end anastomoses techniques. All the renal allografts were placed into retroperitoneum.

Over the years, there have been different surgical techniques described for managing the type of venous drainage and exocrine pancreatic secretions. Enteric drainage of exocrine secretions was performed on all pancreas transplants, systemic venous outflow (postcava), and arterial reconstruction with "y" graft (iliac artery). Lastly in all cases of renal transplant, the artery was anastomosed in all cases. The pancreas was placed intraabdominally in all cases.

Many different antibody induction regimens were noted. For analyses, antibody induction therapy was defined as the use of depleting [e.g., antithymocyte globulin (ATG)] and/ or nondepleting (rituximab, basiliximab) antibodies.

The diagnosis of acute rejection after kidney transplantation was confirmed by a kidney graft biopsy and based on the imaging, clinical, and laboratory, data. When clinically indicated, pancreas graft biopsies were performed. The type of rejection was classified using the Banff score. Kidney graft function was followed by measuring serum creatinine levels and creatinine clearance. For pancreas graft function, serum glycemia, insulin levels, C-peptide, and HbA1c were used.

\section{Statistical analyses}

The primary outcomes of the interest were allograft survival. This was defined as the time between the date of transplantation and/or the date of the graft failure. Failure was marked by a return to dialysis or retransplantation or the last date of follow-up had a functioning graft. Other outcomes of interest were if there were transplantation complications, how long the length of stay was, if there were any delayed graft functions, the causes of graft loss, a recurrence of DM, and if there was an allograft rejection.

Baseline characteristics of the donor, recipient, and transplant characteristics, including immunosuppressive drug schedules at discharge, were compared. All data were expressed as mean \pm standard deviations $(\mathrm{SD})$ of three independent experiments, and comparisons were performed using Student's $t$-tests. Patient survival, graft function, and recurrence of DM were estimated using the Kaplan-Meier method, and log-rank test compared the resulting curves. All $\mathrm{P}$ values were two-sided, and statistical significance was defined as a $\mathrm{P}<0.05$ or $\mathrm{P}<0.01$. Statistical analyses were performed using the SPSS 13.0 software (SPSS Inc., Chicago, IL, USA). 


\section{Results}

\section{Baseline characteristics}

Between September 2016 and May 2019, a final cohort of 63 SPK transplants was included in our study cohort. Table 1 describes the baseline characteristics of the two groups, according to diabetes type. SPK transplants in type 2 recipients $(71 \%)$ were mainly performed in our study cohort. Compared with T1DM recipients, T2DM recipients were older at the time of transplant and the onset of diabetes. Moreover, T2DM recipients had older age, duration of diabetes, and pretransplant dialysis time. Other variables were not found to be different between T1DM and T2DM patients.

\section{Posttransplant complications}

For kidney graft, the mean cold ischemia time was $240 \pm 55$ mins implanted on the right iliac vessels for all cases. The mean cold ischemia time for the pancreas grafts were $380 \pm 110$ mins, which was inserted after the kidney. Table 2 shows the posttransplant complication rates in T1DM and T2DM recipients. Only one patient had delayed renal graft function in T2DM group; five patients with T1DM occurred kidney allograft rejection. Eight patients occurred in the T2DM group and did not differ significantly between the two groups $(\mathrm{P}=0.376)$. Until the end of the analysis, two patients (T2DM) required hemodialysis. These two patients lost their kidney grafts due to antibody-mediated rejection.

After the SPK transplants, insulin requirement was stopped were on the first day in the two groups. The patients had a measurement of long-term blood glucose control. Pancreas complications were similar between groups. Only one patient (T1DM) had to take insulin injections (multiple daily infusions) afterwards. This was due to him losing the pancreatic graft from arterial thrombosis. Also, oral hypoglycemic drugs were used in the other two patients (T2DM). The incidence of acute pancreas allograft rejection was $22.2 \%$ for the entire cohort and it did not differ significantly between both the T1DM and T2DM recipients. In the immediate post-transplant period, 5 for pancreatitis, 1 for the duodenal leak, 1 for pancreatic fistula, and 5 for intestinal obstruction. One T2DM patient died from severe pneumonia (3 months after the transplantation). The last cohort death was due to a T1DM patient having cardiovascular disease 25 months after performing the transplantation.
Overall the 1 and 2 years patient survival rate was: $98.4 \%$ and $96.8 \%$; and overall kidney graft survival was $98.4 \%$ and $96.8 \%$ respectively; while it was $96.5 \%$, and $89.0 \%$, respectively, for pancreas grafts. Patient survival, pancreas graft survivals, and kidney graft survival for each group are shown in Figure 1. No statistically significant difference was seen for any types of diabetes.

\section{Discussion}

There are about 21 million people in the United States that have been diagnosed with diabetes, which represents about $9.3 \%$ of the US population. Additionally, there are thought to be another 8.1 million people who have undiagnosed diabetes. Most of these people were diagnosed as having T2DM, with T1DM accounting for $8-10 \%$ of cases (2). The most severe complication of diabetes is diabetic nephropathy. Many clinical pieces of research have suggested that sustained restoration of glycemic control for the T1DM patients with end-stage renal disease results from SPK transplantation (3-5). There is much less of a consensus as to whether patients with T2DM patients have a similar benefit.

In the present study, we studied the characteristics and outcomes of all of the SPK recipients between Sep 2016 and May 2019 in our organ transplantation center. There are older age and a longer pretransplant dialysis time in T2DM recipients. Furthermore, we haven't found significant differences in blood glucose level and patient survival between T1DM and T2DM recipients. Also, T2DM was not associated with increased graft loss when compared with T1DM recipients.

Though the diagnosis of T2DM was once thought to be a contraindication to SPK transplantation, usefulness has been demonstrated on the chosen subjects with type 2 diabetes (6-8). Light et al. (9) analyzed the results of 10 to 15 years of SPK transplants in a single-center retrospective review from 135 type 1 and type 2 insulin-dependent DM patients. The results from this study revealed that the outcome of a SPK transplant is equivalent no matter the type of diabetes. Margreiter et al. (10) led a singlecenter retrospective review that analyzed 21 T2DM SPK recipients and compared them to a conventional T1DM SPK and T2DM kidney transplantation alone (KTA) control group. A multivariate analysis that had adjusted for any potential variables such as donor/recipient age, presence of secondary diabetic complications, body mass index (BMI) 
Table 1 Baseline recipient, donor, and transplant characteristics

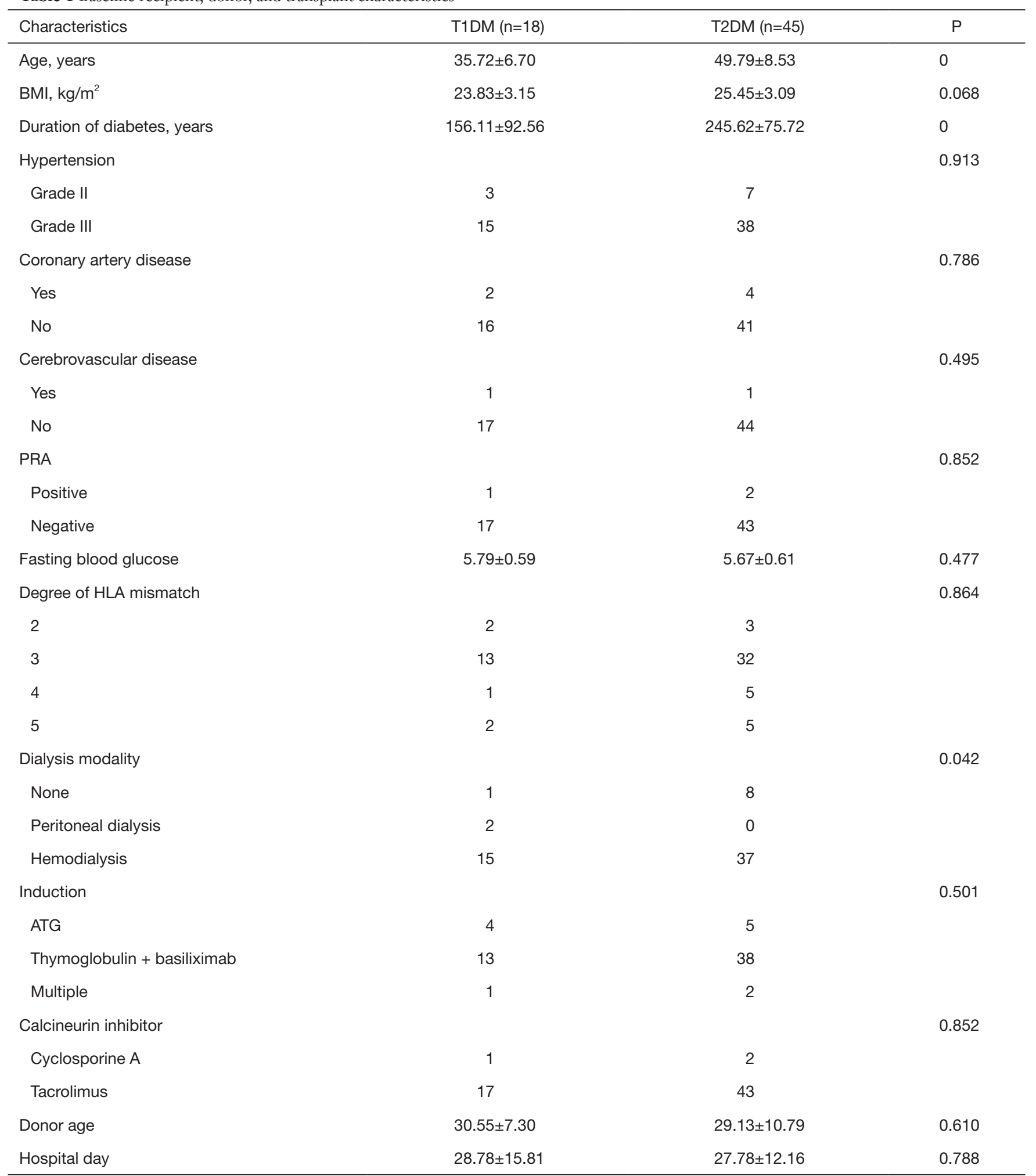

T1DM, type 1 diabetes mellitus; T2DM, type 2 diabetes mellitus; BMI, body mass index; PRA, panel-reactive antibody; HLA, human leukocyte antigen; ATG, antithymocyte globulin. 
Table 2 Post-SPK transplant complications in type 1 and 2 diabetic recipients.

\begin{tabular}{|c|c|c|c|}
\hline Complications & T1DM $(n=18)$ & T2DM $(n=45)$ & $\mathrm{P}$ \\
\hline Delayed graft function & 0 & 1 & 0.524 \\
\hline Kidney rejection & 5 & 8 & 0.376 \\
\hline \multicolumn{4}{|l|}{ Pancreas complications } \\
\hline Pancreas rejection & 5 & 9 & 0.502 \\
\hline Duodenal leak & 0 & 1 & 0.524 \\
\hline Pancreatic fistula & 0 & 1 & 0.524 \\
\hline Portal thrombosis & 2 & 2 & 0.324 \\
\hline
\end{tabular}

SPK, simultaneous pancreas-kidney; T1DM, type 1 diabetes mellitus; T2DM, type 2 diabetes mellitus.

A

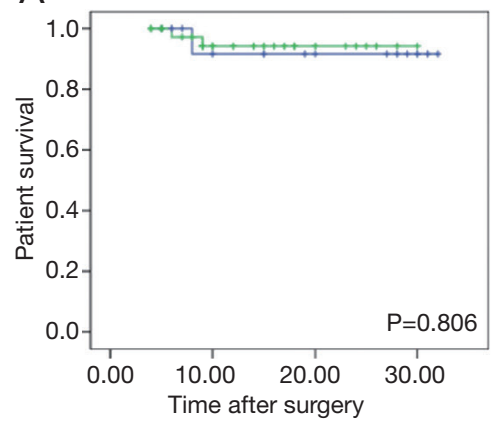

B

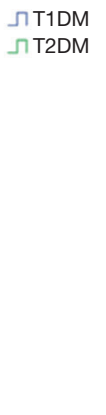

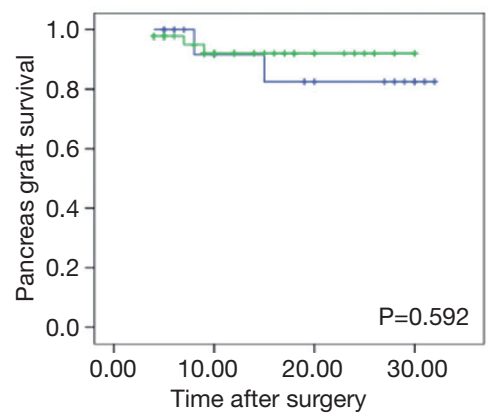

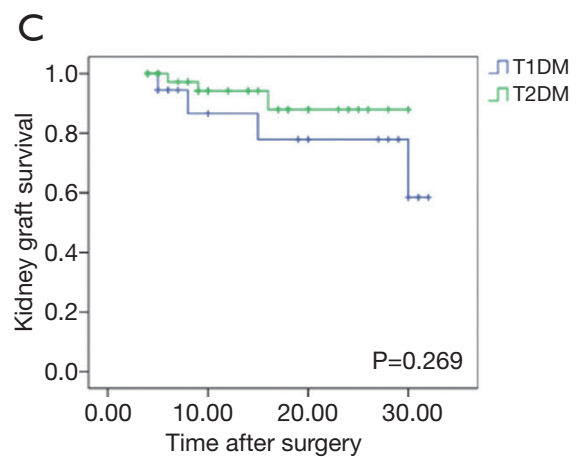

Figure 1 Kaplan-Meier curves for (A) patient survival, (B) overall pancreas graft survival, (C) overall kidney graft survival in type 1 diabetic and type 2 diabetic recipients. T1DM, type 1 diabetes mellitus; T2DM, type 2 diabetes mellitus.

waitlist time, cold ischemic time, delayed graft function, and coronary risk factors. After the analysis was finished it was revealed that there was no statistical significance between the recipients in the T1DM and T2DM groups. A growing body of evidence has shown that in suitably selected patients with T2DM, the clinical course and outcomes following SPK transplants were similar to the parallel patients with T1DM that underwent the SPK transplants $(7,11,12)$.

Diabetes is generally considered as either T1DM or T2DM, depending on many factors. For example: genetic preponderance, age of onset, body habitus, inciting origin, and associated symptoms. However, there is a great amount of overlapping between the clinical symptoms of T1DM and T2DM. This may suggest that DM is actually a heterogeneous disorder, so T1DM and T2DM might have the same roots in insulin resistance that progress in people with a beta-cell failure genetic predisposition. Following decades of debate about the worth of PT for type 2 diabetic patients, the ratio of 1999-2003 pancreas transplant recipients that were classified as having type $2 \mathrm{DM}$ was higher in the SPK $(6 \%)$ than in the pancreas after kidney (PAK) transplantation (4\%) and pancreas transplant alone (PTA) (3\%) categories. For the 1999-2003 SPK recipients, the pancreas graft survival rates (post-transplant insulin independence) 1 year after was not significant in patients with type $1(84.5 \%)$ and type $2(85.2 \%) \mathrm{DM}(13)$.

There are concerns that insulin resistance in T2DM recipients leads a cascade in insulin production with islet exhaustion in a damaging way. This results in allograft failure. Peripheral insulin resistance is associated with 
relative insulin deficiency and insulin secretory defects in T2DM patients (14). Successful SPK transplants achieve endogenous insulin secretion (C-peptide production) that has been shown to be reactive to standard feedback mechanisms. The result is the normalization of the glycosylated hemoglobin levels without needing exogenous insulin administration or close glucose monitoring. Insulin secretion and sensitivity have been shown to improve long term results after SPK for T2DM recipients (15).

The key limitation of the study is the lack of concrete data on the criteria that is used for defining diabetes type. In our study, diabetes type diagnosis in patients with end-stage renal disease relies mainly on medical history and laboratory parameters. Thus, this could lead to an incorrect diagnosis of T2DM. The small size of our study reduced our ability to analyze the subgroup data in our study. Finally, retrospective studies are inadequate in their capability to prove the contributing factors and only show associations.

In conclusion, compared with recipients diagnosed with T1DM, T2DM was associated with similar risks for death and kidney or pancreas allograft survival after an SPK transplant when compared with T1DM recipients. These data provided a framework for identifying best practices to modify recipient selection criteria for SPK transplantation. Further studies should be performed to develop the indications beyond the current acceptable C-peptide levels and BMI.

\section{Acknowledgments}

Funding: This work was supported by the grants from the startup of medical doctor project (No. 52010202-1001).

\section{Footnote}

Conflicts of Interest: The authors have no conflicts of interest to declare.

Ethical Statement: The authors are accountable for all aspects of the work in ensuring that questions related to the accuracy or integrity of any part of the work are appropriately investigated and resolved. The Institutional Review Board of the Second Affiliated Hospital of Guangzhou Medical University approved protocols of the study. Written informed consent was obtained from each participant for the collection of clinical data. This trial is registered with Chinese Clinical Trial Registry, number ChiCTR1900026543.

\section{References}

1. Gruessner AC, Gruessner RW. Pancreas transplantation of US and non-US cases from 2005 to 2014 as reported to the United Network for Organ Sharing (UNOS) and the International Pancreas Transplant Registry (IPTR). Rev Diabet Stud 2016;13:35-58.

2. Dean PG, Kukla A, Stegall MD, et al. Pancreas transplantation. BMJ 2017;357:j1321.

3. White SA, Shaw JA, Sutherland DE. Pancreas transplantation. Lancet 2009;373:1808-17.

4. Dinckan A, Aliosmanoglu I, Kocak H, et al. The impact of method on kidney graft and patient survival in kidneypancreas transplantations for type I diabetes mellitus. Int Surg 2015;100:137-41.

5. Arjona A, Martínez-Cecilia D, Ruiz-Rabelo JF, et al. One hundred one simultaneous pancreas-kidney transplantations: long-term outcomes at a single center. Transplant Proc 2009;41:2463-5.

6. Chakkera HA, Bodner JK, Heilman RL, et al. Outcomes after simultaneous pancreas and kidney transplantation and the discriminative ability of the C-peptide measurement pretransplant among type 1 and type 2 diabetes mellitus. Transplant Proc 2010;42:2650-2.

7. Orlando G, Stratta RJ, Light J. Pancreas transplantation for type 2 diabetes mellitus. Curr Opin Organ Transplant 2011;16:110-5.

8. Stratta RJ, Rogers J, Farney AC, et al. Pancreas transplantation in C-peptide positive patients: does "type" of diabetes really matter? J Am Coll Surg 2015;220:716-27.

9. Light JA, Barhyte DY. Simultaneous pancreas-kidney transplants in type I and type II diabetic patients with endstage renal disease: similar 10-year outcomes. Transplant Proc 2005;37:1283-4.

10. Margreiter C, Resch T, Oberhuber R, et al. Combined pancreas-kidney transplantation for patients with endstage nephropathy caused by type-2 diabetes mellitus. Transplantation 2013;95:1030-6.

11. Nath DS, Gruessner AC, Kandaswamy R, et al. Outcomes of pancreas transplants for patients with type 2 diabetes mellitus. Clin Transplant 2005;19:792-7.

12. Sener A, Cooper M, Bartlett ST. Is there a role for pancreas transplantation in type 2 diabetes mellitus? Transplantation 2010;90:121-3.

13. Hariharan S, Pirsch JD, Lu CY, et al. Pancreas after kidney transplantation. J Am Soc Nephrol 2002;13:1109-18.

14. Wiseman AC, Gralla J. Simultaneous pancreas kidney transplant versus other kidney transplant options in 
patients with type 2 diabetes. Clin J Am Soc Nephrol 2012;7:656-64.

15. Pox C, Ritzel R, Büsing $M$, et al. Combined pancreas and kidney transplantation in a lean type 2 diabetic patient. Effects on insulin secretion and sensitivity. Exp Clin Endocrinol Diabetes 2002;110:420-4.

Cite this article as: Liu L, Xiong Y, Zhang T, Fang J, Zhang L, Li G, Xu L, Lai X, Chen R, Guo Y, Pan G, Ma J, Chen Z. Effect of simultaneous pancreas-kidney transplantation on blood glucose level for patients with end-stage renal disease with type 1 and type 2 diabetes. Ann Transl Med 2019;7(22):631. doi: $10.21037 /$ atm.2019.10.106 